06;07

\title{
Новый тип углеродной наноструктуры на вицинальной поверхности $\operatorname{SiC}(111)-8^{\circ}$
}

\author{
(c) Г.В. Бенеманская ${ }^{1,2}$, П.А. Дементьев ${ }^{1}$, С.А. Кукушкин ${ }^{2-4}$, А.В. Осипов ${ }^{3}$, С.Н. Тимошнев ${ }^{2,5}$ \\ ${ }^{1}$ Физико-технический институт им. А.Ф. Иофффе РАН, Санкт-Петербург, Россия \\ ${ }^{2}$ Институт проблем машиноведения РАН, Санкт-Петербург, Россия \\ ${ }^{3}$ Санкт-Петербургский национальный исследовательский университет информационных технологий, механики и оптики, \\ Санкт-Петербург, Россия \\ ${ }^{4}$ Санкт-Петербургский политехнический университет Петра Великого, Санкт-Петербург, Россия \\ ${ }^{5}$ Санкт-Петербургский национальный исследовательский Академический университет РАН, Санкт-Петербург, Россия \\ E-mail: galina.benemanskaya@mail.ioffe.ru
}

Поступило в Редакцию 4 декабря 2018г.

В окончательной редакции 4 декабря 2018г.

Принято к публикации 5 декабря 2018г.

Впервые исследованы электронные свойства наноинтерфейса $\mathrm{Ba} / \mathrm{SiC} / \mathrm{Si}(111)-8^{\circ}$ методом фотоэлектронной спектроскопии с использованием синхротронного излучения. Эксперименты проведены in situ в сверхвысоком вакууме при субмонослойных покрытиях Ва на образцах $\mathrm{SiC} / \mathrm{Si}(111)-8^{\circ}$, выращенных методом замещения атомов. Обнаружено, что адсорбция Ва вызывает сильные изменения в спектре остовного уровня С $1 s$. Показано, что эффект обусловлен образованием новой, ранее неизвестной углеродной наноструктуры. Установлено, что наноструктура образуется исключительно на вицинальных поверхностях $\mathrm{SiC}$ в присутствии стабилизирующих адсорбированных атомов металла Ва и состоит из углеродных колец, в которых химические связи близки по природе к связям, присущим ароматическим соединениям.

DOI: 10.21883/PJTF.2019.05.47390.17621

Интерес к широкозонному полупроводнику карбиду кремния ( $\mathrm{SiC})$ обусловлен широкими перспективами использования данного материала в полупроводниковых приборах различного назначения. Высокая подвижность электронов, большая величина электрического поля пробоя в сочетании с прекрасными термохимическими характеристиками и высокой радиационной устойчивостью $\mathrm{SiC}$ представляют большой интерес при создании мощных высокотемпературных электронных и оптоэлектронных приборов, работающих на высоких частотах [1]. Несмотря на значительный технологический прогресс в выращивании высококачественных материалов, данных об электронной структуре $\mathrm{SiC}$ недостаточно. Исследования поверхностных состояний и процессов формирования границ раздела металл/SiC имеют особое значение с практической точки зрения. Особенности поверхности $\mathrm{SiC}$ могут оказывать существенное влияние на электронные свойства и таким образом отвечать за благоприятные решения в дальнейшем развитии технологий.

В настоящей работе эпитаксиальные слои $\mathrm{SiC}$ были выращены с помощью оригинального метода замещения атомов подложки. Метод основан на идее химического замещения половины атомов кремния точно таким же числом атомов углерода. Процедура роста подробно описана в работах [2,3]. Укажем ключевые моменты метода. Во время химической реакции в „предкарбидной“ кремниевой фазе образуются пары точечных дефектов $\mathrm{C}$ и вакансий кремния $V_{\mathrm{Si}}$. Эти точечные дефекты расположены строго перпендикулярно направлению [111] в подложке $\mathrm{Si}$. В результате получается очень чистый кар- бид кремния, свободный от примесей фосфора и бора, существующих в исходном кремнии. Важным фактором является приоритетное испарение кремния при росте на ступенчатых (вицинальных) поверхностях и обогащение этих поверхностей углеродом (относительно содержания углерода на сингулярной поверхности).

Фотоэлектронная спектроскопия представляет собой мощный метод изучения электронных свойств и получения наиболее полной информации о зонной структуре материала. Техника отличается высокой чувствительностью к химическим состояниям. Недавно было опубликовано исследование фотоэмиссии различных политипов $\mathrm{SiC}[4,5]$. Однако проблемы, связанные с интерфейсами металл/SiC, остаются слабо изученными. Существует несколько работ по адсорбции металлов Cs [6], Na [7] и Sc [8] на поверхности гексагонального политипа $\mathrm{SiC}$. Недавно была изучена электронная структура интерфейса $\mathrm{Ba} / 3 C-\mathrm{SiC}(111)$ для случая сингулярной поверхности $\mathrm{SiC}$ [9]. Обнаружена заметная модификация спектров остовных уровней $\mathrm{Si} 2 p$ и С $1 s$. Более существенные изменения наблюдались при формировании интерфейса путем адсорбции цезия на вицинальную поверхность $\mathrm{SiC} / \mathrm{Si}(111)-4^{\circ}$ [10]. Было установлено, что при адсорбции атомов Cs на сингулярные поверхности нанослоев $\mathrm{SiC}$ электронная плотность испытывает химический сдвиг от кремния к углероду. Этот результат принципиально отличается от соответствующих данных, полученных для гексагональных кристаллов $\mathrm{SiC}$, выращенных стандартным методом $[11,12]$. При изменении электронной плотности 


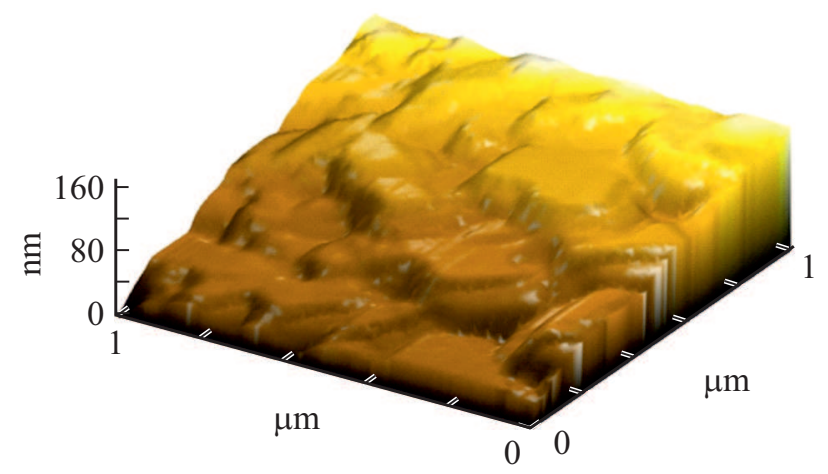

Рис. 1. Трехмерное изображение АСМ области $(1 \times 1 \mu \mathrm{m})$ поверхности $\mathrm{SiC} / \mathrm{Si}(111)-8^{\circ}$.

в кристаллах $\mathrm{SiC}$, выращенных стандартными методами, поверхностная реконструкция приводит к образованию связей $\mathrm{Si}-\mathrm{C}$, отличных от связей в нанослоях $\mathrm{SiC}$. $\mathrm{B}$ нанослоях $\mathrm{SiC}$ часть связей просто обрывается на поверхности. В этом случае атом углерода „перетягивает“ электронную плотность от кремния. Адсорбция атомов Ва или Cs частично восстанавливает равновесное состояние электронной плотности, возвращая атомы $\mathrm{Si}$ и С из возбужденного состояния в состояние, близкое к объемному в пленке $\mathrm{SiC}$.

В настоящей работе исследованы электронные и фотоэмиссионные свойства эпитаксиальных нанослоев $\mathrm{SiC}$, выращенных оригинальным методом на вицинальных поверхностях $\mathrm{Si}(111)$, наклоненных под углами $8^{\circ}$ к плоскости (111). Впервые изучена модификация спектров фотоэмиссии остовных уровней С $1 s$ при поэтапной адсорбции бария на поверхности $\mathrm{SiC} / \mathrm{Si}(111)-8^{\circ}$. С помощью фотоэмиссионной и рамановской спектроскопии обнаружен новый тип двумерной наноструктуры на основе углерода, образующейся при адсорбции Ва. Механизм формирования интерфейса анализируется с использованием экспериментальных и теоретических методов.

Эпитаксиальные нанослои $\mathrm{SiC}$ были выращены оригинальным методом замещения атомов на вицинальных поверхностях $\mathrm{Si}(111)-8^{\circ}$ [2,3]. Метод роста определяет важные факторы: поверхность всегда обогащается C; существенными дефектами являются вакансии Si. Образцы были охарактеризованы методами рентгеновской дифракции и атомно-силовой микроскопии (АСМ). Толщины слоев $\mathrm{SiC}$ составляли 100-150 nm.

На рис. 1 приведено АСМ-изображение морфологии поверхности образца. Видно, что поверхность нерегулярно ступенчата. Высота шага составляет $\sim 10 \mathrm{~nm}$, что намного превышает высоту моноатомного шага. После адсорбции Ва существенного изменения морфологии поверхности не наблюдалось.

Фотоэмиссионные исследования выполнены на синхротроне BESSY II (Берлин, Германия) методом фотоэлектронной спектроскопии при энергиях фотонов в диапазоне $130-450 \mathrm{eV}$. Эксперименты проводились в сверхвысоком вакууме $\sim 5 \cdot 10^{-10}$ Torr при комнатной температуре. Регистрировались фотоэлектроны по нормали к поверхности, возбуждающий пучок падал на поверхность образца под углом $45^{\circ}$. Полное энергетическое разрешение составляло $50 \mathrm{meV}$. Образцы $\mathrm{SiC}$ предварительно отжигались при температуре $\sim 950 \mathrm{~K}$. Атомарно-чистый барий напылялся пошагово на чистую поверхность образца из стандартного источника. Следует отметить, что один монослой (ML) определяется как один полный слой атомов Ва и равен для сингулярной поверхности $\sim 6.25 \cdot 10^{14}$ atoms $/ \mathrm{cm}^{2}$. Реальная поверхность $\mathrm{SiC}$ не является гладкой на атомарном уровне. В общем случае поверхность $\mathrm{SiC}$ содержит выступы и впадины, т.е. реально поверхность является шероховатой, поэтому атомы Ва могут собираться в кластеры. В результате о полном покрытии барием поверхности $\mathrm{SiC}$ можно говорить лишь с некоторой долей осторожности. Степень субмонослойного покрытия оценивалась также по интенсивности фотоэмиссии остовного дублетного максимума Ва $4 d$ (рис. $2, b$ ).

На рис. 2, $a$ представлены спектры остовного уровня $\mathrm{C} 1 s$, полученные для чистой поверхности $\mathrm{SiC}$ (кривая 1) и для интерфейса $\mathrm{Ba} / \mathrm{SiC}$ при различных покрытиях $\mathrm{Ba}$ (кривые 2,3). Найдено, что спектр C $1 s$ для чистой поверхности $\mathrm{SiC}$ состоит из двух мод. Основная мода $B$ соответствует атомам С в объеме образца. Мода $S 1$ при большей энергии связи соответствует атомам углерода, которые расположены на террасах в поверхностном слое над атомами $\mathrm{Si}$ и при взаимодействии образуют двойной слой $\mathrm{C}-\mathrm{Si}$. Похожие спектры можно наблюдать для поверхности $3 C-\mathrm{Si}(111)$ [13]. Положение моды $S 1$ при больших энергиях связи свидетельствует о том, что для чистой поверхности, обогащенной углеродом, в слое $\mathrm{C}-\mathrm{Si}$ происходит увеличение ионности для атомов $\mathrm{C}$.

Крайне необычный спектр C $1 s$ наблюдается при формировании интерфейса $\mathrm{Ba} / \mathrm{SiC}(111)-8^{\circ}$ (рис. 2, $a$, кривые $2,3)$. Найдено, что в спектре появляются две новые моды $S 2$ (сдвиг энергии на $3.8 \mathrm{eV}$ ) и $S U$ (сдвиг энергии $7.0 \mathrm{eV}$ ). Мода $S 1$ сдвигается в сторону больших энергий связи на $\sim 0.3 \mathrm{eV}$. Спектр C $1 s$, лежащий в столь широкой области значений энергии и содержащий столь богатый набор интенсивных мод, наблюдается впервые. Это указывает на структурные особенности вицинальной поверхности нанослоя $\mathrm{SiC}(111)-8^{\circ}$ с террасами и ступенями (рис. 1), а также на необычные электронные и морфологические особенности поверхностных атомов $\mathrm{C}$, образующих связи $\mathrm{C}-\mathrm{Si}$ и гибридизированные связи $s p^{2}$ и $s p^{3}$ в обогащенном углеродом слое $\mathrm{C}-\mathrm{C}$ на поверхности.

Следует заметить также, что пик $S U$, вероятно, является сателлитом типа „встряски“ (shake-up). При этом энергия связи состояния $S U$ совпадает с известным значением энергии связи для сателлита „встряски“ в графене на поверхности $4 H-\mathrm{SiC}(0001)$ [14]. Сателлит $S U$ наблюдается также в спектрах C $1 s$ в других органических соединениях. Важно отметить, что в отличие от 

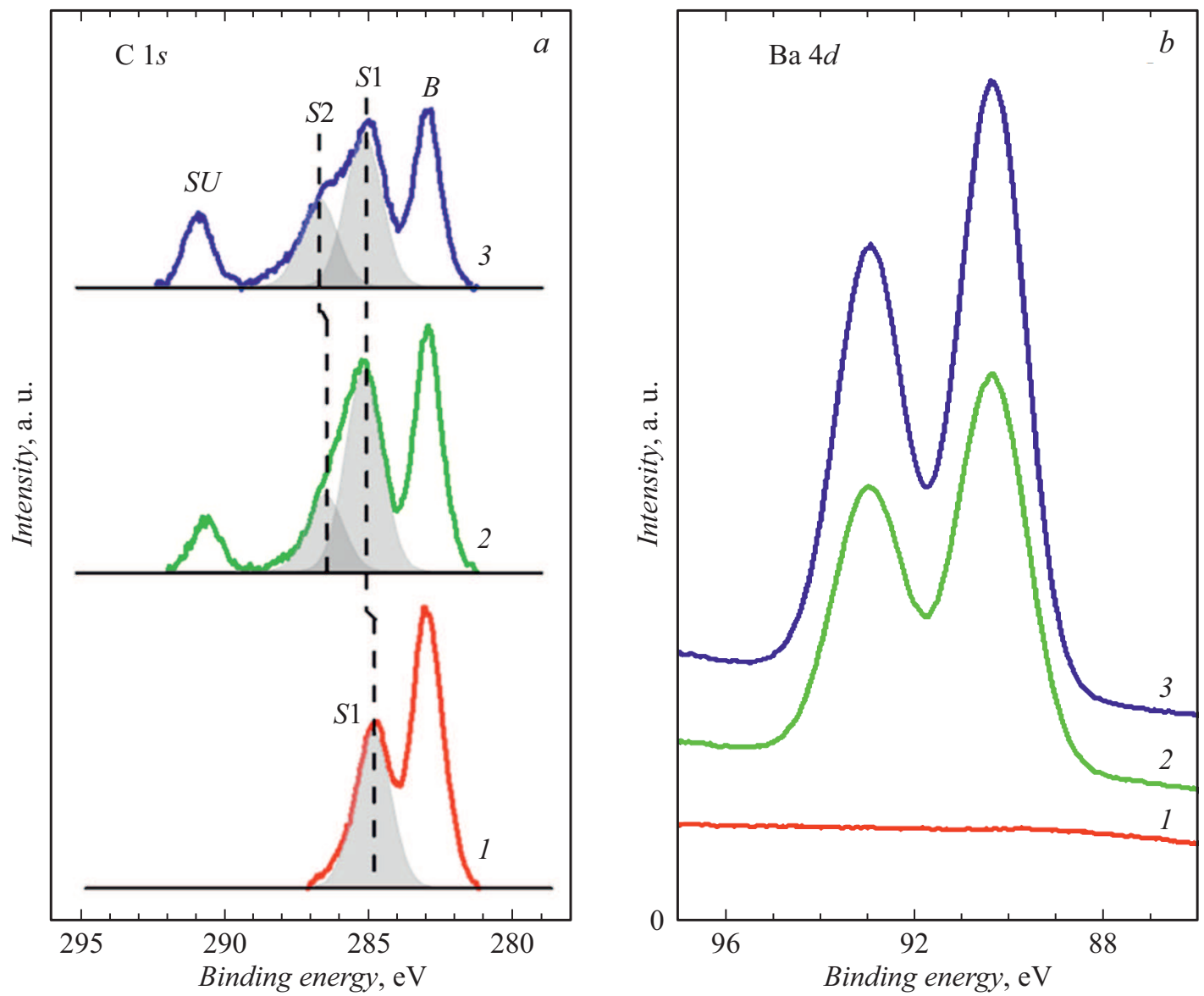

Рис. 2. Фотоэмиссионные спектры остовного уровня C $1 s(a)$ и остовного уровня $\mathrm{Ba} 4 d(b)$ для чистой поверхности $\mathrm{SiC}(111)-8^{\circ}$ (1) и для интерфейса $\mathrm{Ba} / \mathrm{SiC}(111)-8^{\circ}$ при покрытии $\mathrm{Ba} 0.5$ (2) и $1.2 \mathrm{ML}(3)$. Энергия возбуждения $h v=450(a)$ и $130 \mathrm{eV}(b)$.

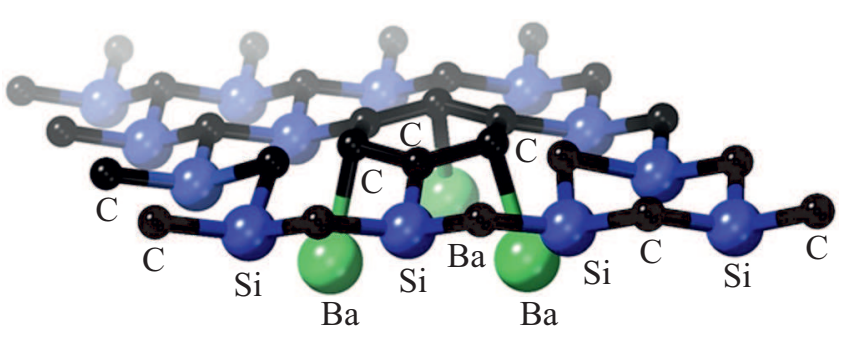

Рис. 3. Схема модификации атомной структуры поверхности $\mathrm{SiC}$ и образования новых углеродных связей при адсорбции $\mathrm{Ba}$.

других систем [14] энергия максимума пика $S U$, как следует из рис. $2, a$, соответствует $292 \mathrm{eV}$. Подобное расположение максимума пика $S U$ на энергетической шкале, как известно, указывает на возможное образование колец углерода, подобных кольцам ароматических соединений, т.е. указывает на возможное образование колец углерода с $s p^{2}$-типом гибридизации. Для получения дополнительной информации о состоянии углеродного слоя на вицинальной поверхности после адсорбции Ва и природе необычного вида спектра С $1 s$ были исследованы рамановский спектр образца $\mathrm{SiC} / \mathrm{Si}(111)$ - $8^{\circ}$ в исходном состоянии и рамановский спектр интерфейса $\mathrm{Ba} / \mathrm{SiC} / \mathrm{Si}(111)-8^{\circ}$ с адсорбированным монослоем Ва. Установлено, что для интерфейса в области спектра $1200-1800 \mathrm{~cm}^{-1}$ появляются новые особенности, которые не наблюдаются в этой области для того же самого слоя $\mathrm{SiC}$, но без Ва. В присутствии атомов Ва появляются две характерные полосы $D$ и $G$. Пик $D$ указывает на формирование $s p^{3}$-связей $\mathrm{C}-\mathrm{C}$, а пик $\mathrm{G}-$ на присутствие $s p^{2}$-связей $\mathrm{C}-\mathrm{C}$. Кроме того, пик $G$ указывает на возможное формирование углеродных колец. Наличие в спектре как пика $G$, так и пика $D$ свидетельствует о сложной комбинации данных связей, возникающих из-за возмущающего воздействия атомов бария. Таким образом, присутствие в рамановском спектре полос $G$ и $D$ с одновременным присутствием пика shake-up в области $292 \mathrm{eV}$ в спектрах фотоэмиссии однозначно указывает на то, что в процессе адсорбции Ва на вицинальных образцах $\mathrm{SiC}$, выращенных методом замещения атомов, образуются кольца углерода, подобные кольцам ароматических соединений.

На рис. 3 представлена схема возможной модификации атомной структуры поверхности $\mathrm{SiC}$ и образования новых углеродных связей при адсорбции Ва. Эффект формирования подобных ароматическим колец на по- 
верхности образцов $\mathrm{SiC}$ является новым и стабильно наблюдается только на вицинальных поверхностях $\mathrm{SiC}-4^{\circ}$, $\mathrm{SiC}-8^{\circ}$ при адсорбции металлов $\mathrm{Ba}, \mathrm{Cs}$.

Авторы выражают благодарность М.Н. Лапушкину за помощь в экспериментальной работе, а также центру Гельмгольца в Берлине за возможность проведения исследований на синхротроне BESSY II.

Исследования поддержаны Российским научным фондом (грант № 14-12-01102).

\section{Список литературы}

[1] Eddy C.R., Jr., Gaskill D.K. // Science. 2009. V. 324. N 5933. P. 1398-1400. DOI: $10.1126 /$ science. 1168704

[2] Кукушикин С.А., Осипов А.В. // ФТТ. 2008. Т. 50. В. 7. C. 1188-1195.

[3] Kukushkin S.A., Osipov A.V. // J. Appl. Phys. 2013. V. 113. N 2. P. 024909 . DOI: $10.1063 / 1.4773343$

[4] Wang J., Zhang L., Zeng Q., Vignoles G.L., Cheng L., Guette A. // Phys. Rev. B. 2009. V. 79. N 12. P. 125304. DOI: 10.1103/PhysRevB.79.125304

[5] Bosi M., Attolini G., Negri M., Frigeri C., Buffagni E., Ferrari C., Rimoldi T., Cristofolini L., Aversa L., Tatti R., Verucchi R. // J. Cryst. Growth. 2013. V. 383. P. 84-94. DOI: 10.1016/j.jcrysgro.2013.08.005

[6] Watcharinyanon S., Virojanadara C., Johansson L.I. // Surf. Sci. 2011. V. 605. N 21-22. P. 1918-1922.

DOI: 10.1016/j.susc.2011.07.007

[7] Watcharinyanon S., Johansson L.I., Xia C., Virojanadara C. // J. Appl. Phys. 2012. V. 111. N 8. P. 083711. DOI: $10.1063 / 1.4704396$

[8] King S.W., Nemanich R.J., Davis R.F. // Phys. Status Solidi B. 2015. V. 252. N 2. P. $391-396$. DOI: $10.1002 /$ pssb. 201451340

[9] Kukushkin S.A., Benemanskaya G.V., Dementev P.A., Timoshnev S.N., Senkovskiy B. // J. Phys. Chem. Solids. 2016. V. 90. P. 40-44. DOI: 10.1016/j.jpcs.2015.10.018

[10] Бенеманская Г.В., Дементьев П.А., Кукушкин С.А., Лапушкин М.Н., Осипов А.В., Тимошнев С.Н. // Письма в ЖТФ. 2016. Т. 42. В. 23. C. 51-57.

DOI: $10.21883 /$ pjtf.2016.23.43982.16389

[11] Virojanadara C., Hetzel M., Johansson L.I., Choyke W.J., Starke U. // Surf. Sci. 2008. V. 602. N 2. P. 525-533. DOI: 10.1016/j.susc.2007.11.012

[12] Kang C., Tang J., Li L., Pan H., Xu P., Wei S., Chen X, Xu X. // Appl. Surf. Sci. 2012. V. 258. N 6. P. 2187-2191. DOI: 10.1016/j.apsusc.2011.02.068

[13] Takahashi R., Handa H., Shunsuke A., Imaizumi K., Fukidome H., Yoshigoe A., Teraoka Y., Suemitsu M. /I Jpn. J. Appl. Phys. 2011. V. 50. N 7R. P. 070103. DOI: 10.1143/JJAP.50.070103

[14] Biedermann L.B., Bolen M.L., Capano M.A., Zemlyanov D., Reifenberger R.G. // Phys. Rev. B. 2009. V. 79. N 12. P. 125411. DOI: 10.1103/PhysRevB.79.125411 